\title{
Relativistic-Klystron Two-Beam Accelerator as a Power Source for Future Linear Colliders
}

\author{
S. M. Lidia, D. E. Anderson, S. Eylon, \\ E. Henestroza, D. L. Vanecek, S. S. Yu, \\ G. A. Westenskow, and T. L. Houck
}

This paper was prepared for submittal to the

RF-98 Workshop

Pajaro Dunes, CA

October 5-9, 1998

October 5, 1998

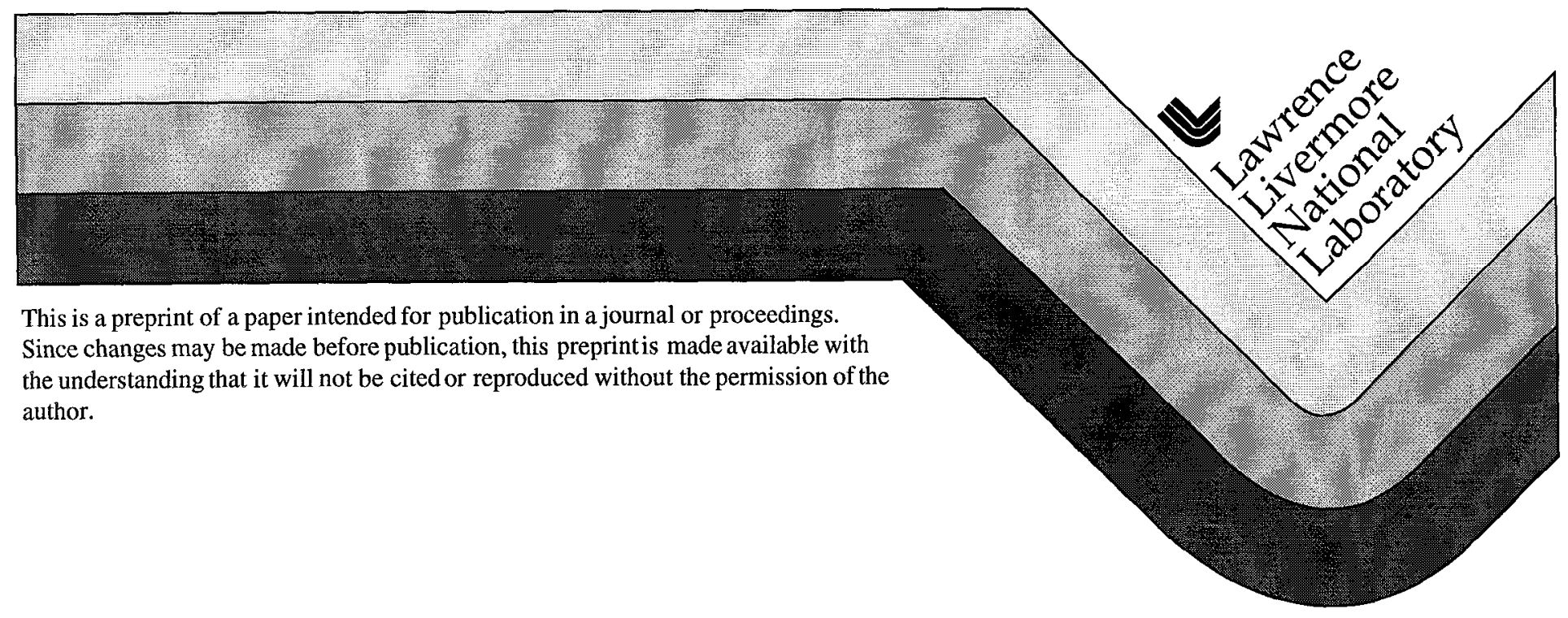




\section{DISCLAIMER}

This document was prepared as an account of work sponsored by an agency of the United States Government. Neither the United States Government nor the University of California nor any of their employees, makes any warranty, express or implied, or assumes any legal liability or responsibility for the accuracy, completeness, or usefulness of any information, apparatus, product, or process disclosed, or represents that its use would not infringe privately owned rights. Reference herein to any specific commercial product, process, or service by trade name, trademark, manufacturer, or otherwise, does not necessarily constitute or imply its endorsement, recommendation, or favoring by the United States Government or the University of California. The views and opinions of authors expressed herein do not necessarily state or reflect those of the United States Government or the University of California, and shall not be used for advertising or product endorsement purposes. 


\title{
RELATIVISTIC-KLYSTRON TWO-BEAM ACCELERATOR AS A POWER SOURCE FOR FUTURE LINEAR COLLIDERS
}

\author{
S.M. Lidia, D.E. Anderson, S. Eylon, E. Henestroza, D.L. Vanecek, S.S. Yu \\ Lawrence Berkeley National Laboratory, Berkeley, CA 94720 USA \\ G.A. Westenskow, T.L. Houck \\ Lawrence Livermore National Laboratory, Livermore, CA 94550 USA
}

\begin{abstract}
The technical challenge for Inaking two-beam accelerators into realizable power sources for high-energy colliders lies in the creation of the drive beam and in its propagation over long distances through multiple extraction sections. This year we have been constructing a $1.2-\mathrm{kA}, 1-\mathrm{MeV}$, induction gun for a prototype relativistic klystron two-beam accelerator (RK-TBA). The electron source will be a $8.9 \mathrm{~cm}$ diameter, thermionic, flat-surface cathode with a maximum shroud field stress of approximately $165 \mathrm{kV} / \mathrm{cm}$. Additional design parameters for the injector include a pulse length of over 150-ns flat top (1\% energy variation), and a normalized edge emittance of less than 300 pi-mm-mr. The prototype accelerator will be used to study physics, engineering, and costing issues involved in the application of the RK-TBA concept to linear colliders. We have also been studying optimization parameters, such as frequency, for the application of the RK-TBA concept to multi-TeV linear colliders. As an rf power source the RK-TBA scales favorably up to frequencies around $35 \mathrm{GHz}$. An overview of this work with details of the design and performance of the prototype injector, beam line, and diagnostics will be presented.
\end{abstract}

\section{INTRODUCTION}

Induction accelerators are a unique source for high-current, high-brightness, electron beams. A collaboration between the Lawrence Livermore National Laboratory (LLNL) and Lawrence Berkeley National Laboratory (LBNL) has been studying if power sources based on the Relativistic Klystron Two-Beam Accelerator (RK-TBA) concept for several years $[1,2]$. Demanding beam parameters are required of the electron source, an induction injector, to achieve the transport goals. A test facility, called the RTA, has been established at LBNL [3] to verify the analysis used in the design study. The primary effort of the facility is the construction of a prototype RK-TBA subunit that will permit the study of technical issues, system efficiencies, and costing. In this paper, we will discuss the development of the RTA electron source and it's pulsed power system, which has recently been constructed and is now undergoing testing. Figure 1 is a photograph of the gun undergoing initial tests. Beam tests will be performed this fall. 


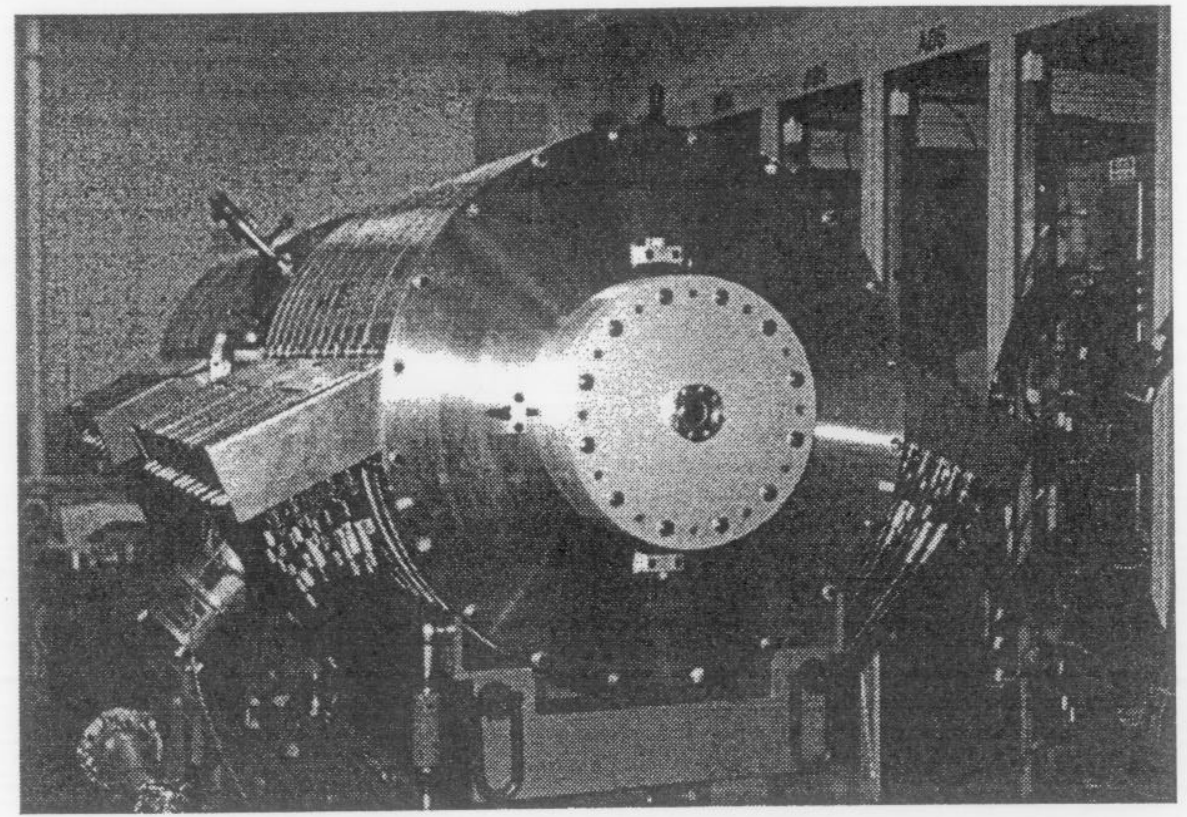

Figure 1. RTA gun

\section{RTA GUN}

A major part of our effort during the past year has been towards the design of a low emittance electron source for the RTA accelerator. We expect to produce an electron source with a much lower emittance than typical induction guns. The electron source will be a 8.9-cm-diameter, thermionic, flat-surface M-type cathode with a maximum shroud field stress of approximately $165 \mathrm{kV} / \mathrm{cm}$. An emission density of $20 \mathrm{~A} / \mathrm{cm}^{2}$ is required from the cathode to produce a $1.2-\mathrm{kA}$ beam.

The RTA gun has 72 induction cores, each driven at $14 \mathrm{kV}$. The voltage from the cores are summed across the A-K gap to produce $1 \mathrm{MV}$. The cells are segmented radially to reduce the individual aspect $(\Delta \mathrm{r} / \Delta \mathrm{z})$ ratio of the cores. The lower aspect ratio reduces the variation in core impedance during the voltage pulse simplifying the pulse forming network (PFN) design.

We have done high-voltage tests on the gun. In operation a $500 \mathrm{kV}$ potential is developed across each of the two 30-cm-ID PYREX insulators producing a $5.1 \mathrm{kV} / \mathrm{cm}$ average gradient along the insulator. The maximum fields at the triple points, the intersection of insulator, vacuum, and metal, is less than $3.5 \mathrm{kV} / \mathrm{cm}$. Maximum surface 
field in the cathode stalk of the gun is about $85 \mathrm{kV} / \mathrm{cm}$. The maximum field is about 116 $\mathrm{kV} / \mathrm{cm}$ on the anode stalk.

Initial beam focusing in the gun is accomplished by large-bore air-core solenoids installed on the central pumping spool. The first solenoid is operated to null the magnetic field from the other solenoids at the cathode front surface. With a flat cathode a high $\mathrm{dB} / \mathrm{dz}$ is needed to prevent the beam from hitting the anode surfaces. The magnetic field at a distance of $20 \mathrm{~cm}$ from the cathode is about 650 gauss. There are seven smaller solenoids located within the anode stalk to provide additional focusing to transport the beam to the end of the gun.

\subsection{Pulsed Power System for the RTA Gun}

The pulsed power system for the gun consists of a $20-\mathrm{kV}$ High-Voltage Power Supply, 6-kJ Energy Storage Bank, two Command Resonant Charging (CRC) Chassis, 24 Switched Pulse Forming Networks, and four Induction Core Reset Pulsers. Each PFN will drive a single 3-core induction cell of the gun.

Segmenting the core in the induction cell and driving the individual core segments avoids a high-voltage step-up transformer. This reduces the developmental effort needed to achieve a "good" flattop pulse (minimal energy variation) with fast risetime and improves the efficiency of the overall pulsed power system. Our system of low-voltage PFNs driving multiple core induction cells is similar to the system envisioned for the full scale RK-TBA design. For the gun core material we chose 20 -mm-thick $2605 \mathrm{SC}$ METGLAS. In the RTA main extraction section we will use a lower loss 2714AS METGLAS for the induction cores.

Design of the switched PFNs follows easily from published METGLAS core loss data [4]. For the RTA induction cells, a flux swing of $2.6 \mathrm{~T}$ in $400 \mathrm{~ns}$ (FWHM) results in a magnetization rate of $6.5 \mathrm{~T} / \mu \mathrm{s}$. At this rate, a loss density of $1800 \mathrm{~J} / \mathrm{m}^{3}$ translates into $30 \mathrm{~J}$ lost in a cell with $16.7 \times 10^{3} \mathrm{~cm}^{3}$ of 2605SC METGLAS. For a cell input voltage of $14 \mathrm{kV}$ applied for $400 \mathrm{~ns}$, these losses require that $5900 \mathrm{~A}$ be supplied to the three radial cores. An additional $3600 \mathrm{~A}$ is required to supply beam current $(1200 \mathrm{~A} \times 3 \mathrm{cores} / \mathrm{cell}$ ), resulting is a total current of $9 \mathrm{kA}$. The required drive impedance for a cell is then $1.5 \Omega$, which is provided by the PFN module.

An area of concern was the variation of the energy loss for different METGLAS cores. Experience [5] with the 72 cores in the gun leads us to believe that for a large RKTBA, matching cell cores should permit acceptable energy loss variation.

\subsection{Gun Voltage Waveform}

Achieving the fast risetime necessary to minimize the volt-seconds required for the injector cores presented a challenge. Budget constraints coupled with the large availability of EEV CX1538 thyratrons from the ATA program at LLNL made these tubes an attractive option. However, their poor lime rate of current change $(4 \mathrm{kA} / \mu \mathrm{s}$ rating) made them questionable for this application, which requires about $40 \mathrm{kA} / \mu \mathrm{s}$. A variety of techniques were tried to decrease the risetime. In a $1.5 \Omega$ system, stray circuit inductances must be maintained at or below $100 \mathrm{nH}$ to achieve a $10-90 \%$ risetime of 150 ns. This was accomplished by placing the thyratron between two current sheets 
connecting the PFN output to the output cables. The ionization time of the thyratron was substantially reduced by applying a 1-2 A pre-pulse to the keep-alive grid $300-400 \mathrm{~ns}$ prior to the arrival of the main control-grid pulse. Faster risetimes were achieved with Triton F-130 ceramic thyratrons. An upgrade of the current thyratrons in the gun pulsed power system should allow us to achieve the design 100-ns risetime.

At the 1-MV, 1.2-kA operating conditions we hope to produce a $\pm 1 \%$ gun voltage flat waveform for $150 \mathrm{~ns}$. We will need to adjust the number of turns in appropriate sections of individual PFNs to achieve this goal. Insertion of ferrite material in the center of the inductor coils will allow additional small corrections to the waveform.

\subsection{Diagnostics for the RTA Gun}

A variety of diagnostics [6] will be used to determine the performance of the gun, both permanently installed monitors for general operations and temporary diagnostics specific to commissioning and troubleshooting. Planned diagnostics include an isolated cathode with resistive divider for direct measurement of current emission, resistive-wall and magnetic probe current monitors for measuring beam current and centroid position, capacitive probes for measuring A-K gap voltage, an energy spectrometer, and a pepperpot emittance diagnostic.

The majority of the diagnostics will be installed after the gun. The first $1.4 \mathrm{~m}$ of beam line after the gun will include two beam position and current monitors to allow the offset and angle of the beam at the exit of the injector to be measured. One of these beam position monitors will incorporate a new diagnostic [7] based on the optical Pockels effect [8] to measure the peak electric field strength at the beam-tube radius in the presence of the beam. A pop-in probe will be incorporated with a pumping port to allow viewing of the transverse beam profile.

The current density will be measured using Cerenkov and/or optical transition radiation from intercepting foils. A primary concern with using foils is possible damage from beam energy deposition. Average heating of the foil can be controlled by adjusting the repetition rate of the injector. The difficulty is the single shot heating where material can be melted and ejected before the heat is conducted away. As an example, to avoid damage for a thin quartz foil, the beam diameter must be larger than $2 \mathrm{~cm}$ for a $1-\mathrm{kA}$, 300 -ns, relativistic electron beam. The significant levels of energy deposited in the foil could affect the dielectric constant or generate surface plasma that could be confused with a variation in beam parameters.

Three different methods may be used to determine the A-K voltage and beam energy. The first method involves measuring the applied voltage to the induction cores at the connection of the power fecds to the induction cells. Capacitive $\mathrm{dV} / \mathrm{dt}$ pickup probes are used for a more direct measurement of the A-K gap voltage and also to provide greater bandwidth with respect to the resistive dividers. We also hope to employ a conventional energy spectrometer comprised of an on-axis collimator, dipole magnet, scintillator, and viewing port to directly measure the beam energy.

\subsection{Emittance measurement for the gun}

We plan to use a pepperpot emittance diagnostic to help characterize the beam. Measuring the beam emittance is expected to be very difficult as the beam is highly space .... 
charged dominated. Our aperture plate will consist of a rectangular pattern of $121(11 \times 11)$ $250-\mu \mathrm{m}$ apertures with $7 \mathrm{~mm}$ spacing on a $500-\mu \mathrm{m}$ thick tungsten plate. The emittance term that is responsible for spot size growth of the beamlets after the aperture plate is approximately an order of magnitude larger than the space charge term.

Another serious problem concerns the effect of the conductive aperture plate on the beam. It has been demonstrated [9] that, while the local distribution in phase space can be determined, the global $x-x^{\prime}$ curve is dominated by the non-linear focusing of the aperture plate. EGUN simulations performed for our beam parameters indicate that the beam emittance determined from the pepperpot data could be as much as six times the actual emittance in the absence of the aperture plate. This effect can be accounted for in the analysis. However, the accuracy in the final emittance value will suffer.

\section{ACCELERATOR SECTION DESIGN}

Design of the full-scale RK-TBA system has an accelerator section after the gun that raises the beam energy from $1 \mathrm{MeV}$ to $2.5 \mathrm{MeV}$ before starting to bunch the beam at 11.4 GHz. In the full-scale RK-TBA system we expect to use a pulse power system similar to that demonstrated in the gun system for this section. The accelerator cells will be segmented to reduce the required drive. However, to reduce RTA's cost we are planning on using a $120-\mathrm{kV}$ MARX spark-gap pulsed power system to drive 16 unsegmented accelerator cells in this section of RTA. The design is not acceptable for the full-scale machine because of the high gap erosion rates. The spark-gap system can provide a faster risetime than the thyratron system, which will reduce the required cross sectional area of the induction cores. The volt-second rating of the accelerator cores (operated at $120 \mathrm{kV}$ ) will limit the flat top of the beam pulse in the RTA. Installation of additional cells (future upgrade), and operating all the cells at a lower voltage, will increase the useful duration of the current pulse. We also plan to test a High-Gradient Insulator [10] in the first of these accelerator cells. If successful we will construct the 16 cells for RTA accelerator section using High-Gradient Insulators.

\section{STUDIES IN KA-BAND}

To complement the ongoing experimental work for an $11.424-\mathrm{GHz}$ source, we will conduct tests of rf output structures and bunched beam transport at $30-35 \mathrm{GHz}$. This frequency band has been deemed useful for producing the peak power necessary to drive accelerating structures for a multi-TeV collider system. This work is conducted through a collaboration of LBNL personnel with groups at CEA/CESTA and CERN.

\subsection{Tests on LELIA}

Since 1995, the induction linac LELIA at the CEA/CESTA facility has been used to produce a $2-\mathrm{MeV}, 800-\mathrm{A}, 60-\mathrm{ns}$ beam modulated at $35 \mathrm{GHz}$ by a free-electron laser. This work has been conducted with the support of CERN to study the generation of a suitable drive beam to power CLIC Transfer Structures (CTS). Of equal interest is the possibility of using the modulated beam to drive inductively detuned if structures in an RK-TBA. In this case, the rf properties of the cavities determine the longitudinal beam dynamics. Hence, measurements of longitudinal phase space bunching are important. Diagnostics 
techniques developed at CESTA have, for the first time, enabled us to directly view the electron bunching, and to capture the image with a streak camera $[11,12]$.

\subsubsection{Inductively Detuned SW RF Cavities}

The first set of experiments will study the interaction of the modulated beam with standing wave if cavities. Three different cavities are to be constructed and studied sequentially, one idler and two single-output cavities. We have modeled these structures with the Superfish, URMEL-T, and GdfidL codes [13]. The characteristics of the cavities are listed in Table 1.

\begin{tabular}{|c|c|c|c|}
\hline & Idler & Low Q & High Q \\
\hline $\mathrm{f}[\mathrm{GHz}]$ & $34-36$ & 35 & 35 \\
\hline $\mathrm{Q}$ & 363 & 6 & 45 \\
\hline $\mathrm{R} / \mathrm{Q}[\Omega]$ & 45 & 45 & 45 \\
\hline $\mathrm{P}_{\text {out }}[\mathrm{MW}]$ & - & 0.7 & 5.0 \\
\hline $\begin{array}{c}\mathrm{E}_{\text {peak }} \\
{[\mathrm{MV} / \mathrm{m}]}\end{array}$ & 400 & 7 & 50 \\
\hline $\mathrm{M} / \mathrm{m}$ & & & \\
\hline
\end{tabular}

Table 1. Parameters of rf cavities.

The idler cavity has been designed to accept variable-radius 'tuning rings' in the inner pillbox region. These rings adjust the inner radius of the cavity to permit tuning the fundamental mode frequency over the range $34-36 \mathrm{GHz}$. The frequency of the cavity can then be adjusted so that the longitudinal impedance seen by the beam is resonant or detuned (capacitively/inductively). The idler cavity assembly is shown in Figure 2.

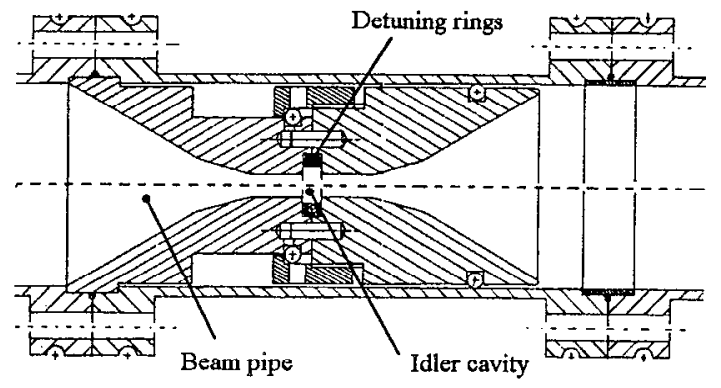

Figure 2. Idler cavity assembly.

The tuning rings will be manufactured by wrapping a layer of stainless steel (304SS) around a narrow spool that exhibits a slight taper. Individual rings will then be cut from this spool. This permits an accurate measure of the ring's inner radius, as well as differences in the radii between different rings.

The output cavities are designed with only a single output port. This port is attached to a connecting waveguide, which is expanded to mate with standard WR-28 guide. Figure 3 shows a quarter of the geometry of the structure. 


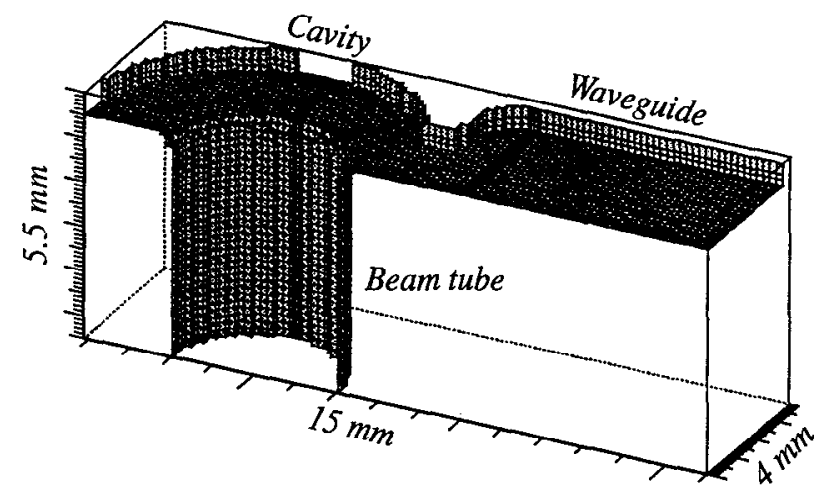

Figure 3. Output cavity quarter-geometry. The beam travels vertically through the beam tube.

This single output port can introduce an unwanted transverse impulse to the beam as it traverses the structure. The pillbox region of the cavity has been designed slightly offcenter from the beamline axis to compensate for this. As a result, the linear variation of the longitudinal electric field has been strongly suppressed. A small quadratic variation is still present. However, any quadrupole interaction is slight. A cross-section of the longitudinal electric field distribution in the cavity midplane normal to the beam axis is shown in Figure 4.

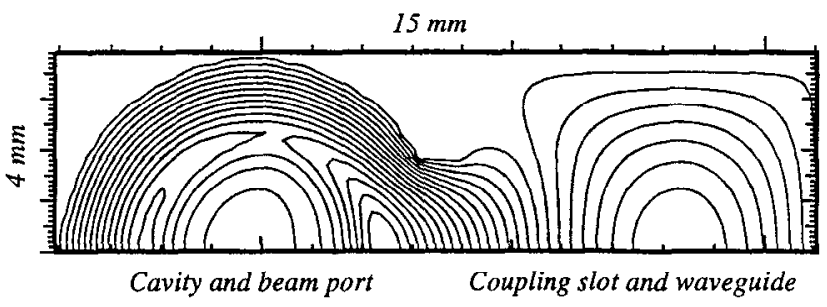

Figure 4. Cross-section of output cavity, showing symmetrization of the longitudinal electric field.

The expected performance of these cavities is listed in Table 1 . As can be seen, only a modest amount of output power is expected from these structures. These first experiments will concentrate mostly on beam dynamics issues: generation and transport of an intense, modulated beam through a narrow aperture cavity; and observation of the interaction of the cavity upon the beam. Later experiments may involve more sophisticated cavity designs, intended to produce rf output levels sufficiently high to drive accelerating structures.

\subsubsection{Longitudinal Beam Dynamics Studies}

Optical measurements will be performed to study the time-dependent beam-cavity interaction. Bunches will be extracted, and their longitudinal bunching characteristics 
measured. This allows us to make a comparison between our simulation codes and experiment. The primary measurement will be of the 'bunching parameter' of the beam before and after it exits the cavity region. This will be compared with measurements of bunching when the beam is freely propagating. Simulation results of the evolution of the bunching parameter along the beamline following the FEL are shown in Figure 5. The cavity is located $28.5 \mathrm{~cm}$ from the end of the FEL. Measurements will also be made of the relative phase of the output rf power with respect to the FEL output.

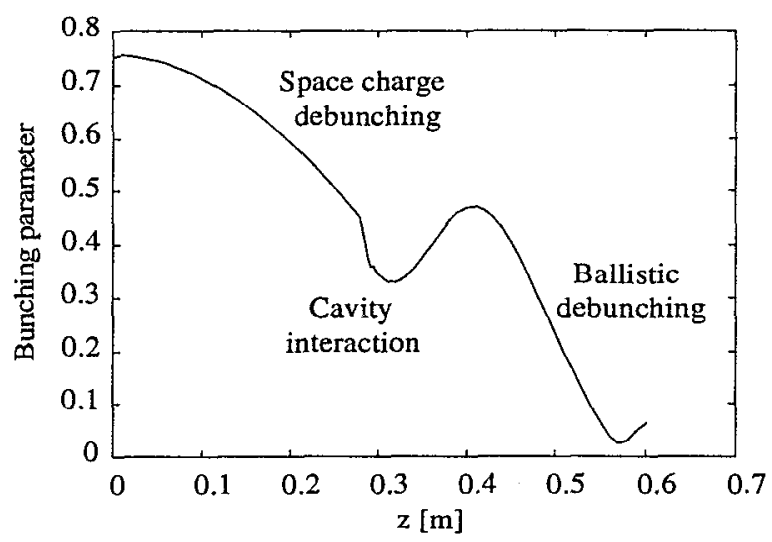

Figure 5. Evolution of the bunching parameter from the end of the FEL through the cavity.

\subsubsection{Observation Of Bunching Characteristics}

An optical diagnostic based on Cerenkov emission will be used to measure the bunching characteristics. The beam is stopped by a movable, fused-silica target. A gated CCD camera and a fast streak camera (2-ps resolution) will be used to collect and analyze a small part of the visible Cerenkov light. Figure 6 shows an example of the streak camera output, clearly displaying the bunching.

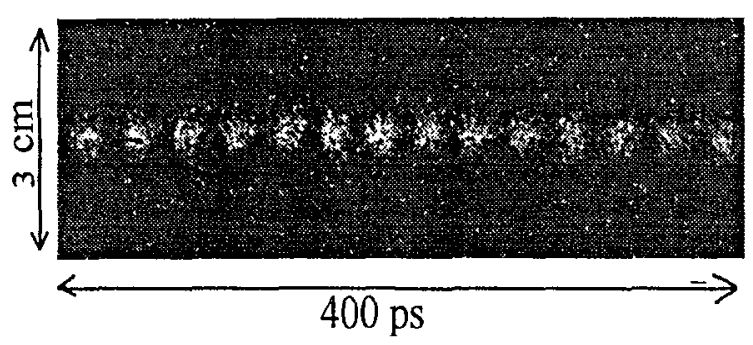

Figure 6. Streak camera image of 35-GIIz bunches.

\subsection{Tests on PIVAIR}

The PIVAIR induction linac generates a nominal 7.2-MeV, 3-kA, 60-ns beam. These values make it very attractive as an injector for a $30-\mathrm{GHz}$ RK-TBA system to power a 
multi-TeV linear collider. A preliminary point design for a multi-TeV-scale linear collider system using an RK-TBA driver and operating at $30 \mathrm{GHz}$ has already been presented [14]. The RK-TBA drive bean architecture is shown in Figure 7.

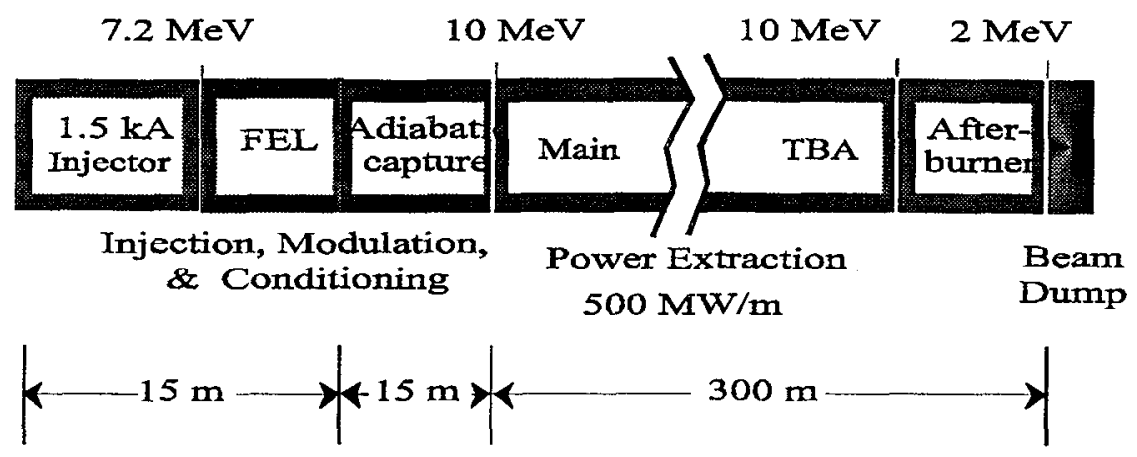

Figure 7. 30-GHz RKTBA architecture.

The front end of the drive beam injector for the Main TBA is composed of an electron gun and accelerator ('Injector'), a free electron laser ('FEL') to provide the modulation, and an 'Adiabatic capture' section to provide for bunch compression, additional acceleration, and other pulse conditioning. This latter effect may include shaping of the front-end current profile to provide a ramped current pulse. Power extraction and reacceleration then occurs in the Main TBA section.

A current proposal [15] seeks to use PIVAIR for TBA-related studies. An FEL to provide $30-\mathrm{GHz}$ modulation will be constructed, followed by a beamline to support TBA studies. Thus PIVAIR, operated as a test stand, will provide a valuable resource in the effort to examine beam dynamics and to test designs of beamline components for a high frequency RK-TBA. Of particular concern are the if output structures and induction modules in the Main TBA section. Prototypes may be designed and then tested on the PIVAIR beamline, once a modulated beam is present.

\section{ACKNOWLEDGEMENTS}

The work was performed under the auspices of the U.S. Department of Energy by LLNL under contract W-7405-ENG-48, and by LBNL under contract AC03-76SF00098. We thank Andy Sessler and Swapan Chattopadhyay for their support and guidance and thank Wayne Greenway, William Strelo, Bob Benjegerdes and Bob Candelario for their excellent technical support.

\section{REFERENCES}

[1] Sessler, A.M. and Yu, S.S., "Relativistic Klystron Two-Beam Accelerator," Phys. Rev. Lett. 54, 889 (1987). 
[2] Westenskow, G.A., and Houck, T.L., "Relativistic Klystron Two-Beam Accelerator," IEEE Trans. on Plasma Sci., 22, 750 (1994).

[3] Houck, T.L., and Westenskow, G.A., "Prototype Microwave Source for a Relativistic Klystron Two Beam Accelerator" IEEE Trans. on Plasma Sci., 24, 938 (1996).

[4] Smith, C.H., and Barberi, L., "Dynamic Magnetization of Metallic Glasses," in Proc. of the 5th. IEEE Int'1 Pulsed Power Conf., 1985.

[5] Westenskow, G.A., et al., "Relativistic Klystron Two-Bcam Accelerator Studies at the RTA Test Facility", Proc. of the 1997 Particle Accelerator Conference, Vancouver, Canada, 1997.

[6] Houck, T.L., et al., "Diagnostics for a 1.2-kA, 1-MV Electron Induction Injector," Proc. of the 8th Beam Instrumentation Workshop, SLAC, 1998.

[7] Mike Brubaker, Los Alamos National Laboratory, private communication.

[8] Hebner, R.E., et. al., Proceedings of the IEEE, 65, 1524-1548 (1977).

[9] Hughes, T.P., Carlson, R.L., and Moir, D.C., J. Appl. Phys. 68, 2562-2571 (1990).

[10] Houck, T.L., et al., "Stacked Insulator Induction Accelerator Gaps", Proc. of the 1997 Particle Accelerator Conference, Vancouver, Canada, 1997.

[11] J. Gardelle, et. al., Phys. Rev. Lett. 76, 4532 (1996).

[12] J. Gardelle, et. al., Phys. Rev. Lett. 79, 3905 (1997).

[13] K. Halbach and R. Holsinger, Part. Acc. 7, 213 (1976); T. Weiland, Nucl. Inst. Meth. Phys. A216, 329 (1983);W. Bruns, IEEE Trans. Magn., 32, n. 3, (1996).

[14] S. Yu, "30-GHz, 200-MV/m Relativistic Klystron Two-Beam Accelerator," Proceedings of VII International Workshop on Linear Colliders, Zvenigorod, Russia, 1997.

[15] J. Gardelle, et. al., "Proposal to use PIVAIR as a 30-GHz High-Power Generator," Proc. of the XIX International Linear Accelerator Conference, Chicago, USA, 1998. 